\title{
ON THE TOPOLOGY OF SELF-DUAL 4-MANIFOLDS
}

\author{
CLAUDE LeBRUN ${ }^{1}$
}

\begin{abstract}
Obstructions are obtained for the problem of finding a Riemannian metric with self-dual conformal curvature and nonnegative scalar curvature on a given smooth compact 4-manifold. A list is given of those simply connected manifolds conceivably admitting such metrics.
\end{abstract}

Which smooth compact oriented 4-manifolds admit Riemannian metrics with self-dual Weyl curvature? The question has received new impetus from the exciting recent result of Poon [1] that $\mathbf{C P}_{2} \# \mathbf{C P}_{2}$ (i.e. two complex projective planes joined by a hose) has such a metric.

Since the problem is conformally invariant, it is convenient to multiply the metric by a conformal factor so that the scalar curvature becomes constant; the sign of this constant is an invariant of the conformal structure, and we will call a self-dual conformal class "type positive", "type zero", or "type negative", depending on the sign. The fact that such a rescaling of the metric is possible is very nontriival; it was only recently proved by Schoen [2] after having been known for some years as the "Yamabe problem".

Now concerning type negative manifolds little seems to be known, though one may produce examples by taking quotients of hyperbolic space or the complex ball $\mathrm{SU}(2,1) / \mathrm{U}(2)$ by discrete groups. Fortunately, one can say a great deal about the other two cases. Indeed, one has, to begin with, the following:

Proposition 1. Let $M$ be a compact 4-manifold with self-dual Weyl curvature and nonnegative scalar curvature. Then one of the following holds:

(1) the intersection form on $\mathrm{H}^{2}(\mathrm{M})$ is positive definite; or

(2) the scalar curvature is zero and $M$ is a Kähler manifold with the conjugate orientation.

Proof. If alternative (1) fails, there is a nonzero anti-self-dual 2-form $\omega$ satisfying $d \omega=0$; this follows from the fact that de Rham cohomology is represented by harmonic forms (Hodge theory). But the Weitzenböck formula asserts that

$$
\left(d+d^{*}\right)^{2}=\nabla^{*} \nabla-2 C+R / 3
$$

Received by the editors August 28, 1985 and, in revised form October 31, 1985.

1980 Mathematics Subject Classification (1985 Revision). Primary 53C20, 53C55; Secondary 14J28, $57 R 15$

${ }^{1}$ Work partially supported by NSF grant no. DMS 8405956. 
on 2-forms, where $C$ and $R$ are the Weyl and scalar curvatures. Hence

$$
\begin{aligned}
0 & =\int_{M}\left\langle\omega,\left(d+d^{*}\right)^{2} \omega\right\rangle d \text { vol } \\
& =\int\left(\left\langle\omega, \nabla^{*} \nabla \omega\right\rangle-2 C(\omega, \omega)+\frac{R}{3}\|\omega\|^{2}\right) d \text { vol } \\
& =\int\left(\|\nabla \omega\|^{2}-2 C^{-}(\omega, \omega)+\frac{R}{3}\|\omega\|^{2}\right) d \text { vol } \\
& =\int\|\nabla \omega\|^{2} d \text { vol }+\int \frac{R}{3}\|\omega\|^{2} d \text { vol, }
\end{aligned}
$$

where $C^{-}=0$ is the anti-self-dual part of the Weyl curvature. Since $R \geqslant 0$, both terms are nonnegative and so vanish; $\nabla \omega \equiv 0$ and $R \equiv 0$. Since any anti-self-dual real 2-form looks like $\alpha\left(e^{1} \wedge e^{2}-e^{3} \wedge e^{4}\right)$ in a suitable oriented orthonormal frame, if we multiply the metric by a constant, and thereby make $\|\omega\| \equiv 2, \omega_{a}^{b}$ will become a covariantly constant orthogonal almost-complex structure. By the Newlander-Nirenberg theorem, this almost-complex structure is integrable. $M$ is now a Kähler manifold, but equipped with the nonstandard orientation. Q.E.D.

Well, what can be said about alternative (2)? To begin with, one has the following local result:

Proposition 2. Let $M^{4}$ be a Kähler manifold with the conjugate orientation. Then $M$ is self-dual iff the scalar curvature vanishes.

Proof. Since the result is local in character, we may assume that $M$ is spin-i.e. that the canonical bundle $K$ has a square-root $K^{1 / 2}$. Then [3] the primed spin bundle $S_{-}$is $K^{1 / 2} \oplus K^{-1 / 2}$, and the spinor connection is the obvious one. Since the curvature of $K$ is $-2 i R_{c a} \omega_{b}^{c}$, which is a form of type $(1,1)$, requiring that $R \equiv 0$ makes the "Ricci form" orthogonal to $\omega$ and hence self-dual; $S_{-}$is then self-dual. But the anti-self-dual curvature of $S_{-}$is $X_{A^{\prime} B^{\prime} C^{\prime}}^{D^{\prime}} \varepsilon_{A b}$, or $\psi_{A^{\prime} B^{\prime} C^{\prime}}^{D_{A B}^{\prime}}$ given that $R \equiv 0$. Thus it follows that $C_{a b c d}^{-}=\psi_{A^{\prime} B^{\prime} C^{\prime} D^{\prime}} \varepsilon_{A B^{\prime}} \varepsilon_{C D}$ must vanish.

Conversely, if $M$ were self-dual with nonzero scalar curvature, the anti-self-dual curvature of $S_{-}$would be $\Lambda\left(\varepsilon_{A^{\prime} C^{\prime}} \varepsilon_{B^{\prime}}^{D^{\prime}}+\varepsilon_{B^{\prime} C^{\prime}} \varepsilon_{A^{\prime}}{ }^{D^{\prime}}\right) \varepsilon_{A D^{\prime}}, \Lambda \neq 0$. Contracting this with an arbitrary 2-form with typical anti-self-dual part $\chi^{A^{\prime} B^{\prime}} \varepsilon^{A B}$, we conclude that $4 \Lambda \chi_{C^{\prime}}{ }^{D^{\prime}}$ is in the Lie algebra of the holonomy group of $S_{-}$, which therefore has dimension at least (and hence equal to) 3. But for a conjugate-oriented Kähler manifold the holonomy of $S_{-}$must be a subgroup of $U(1)$, showing, by contradiction, that for such a manifold to be self-dual one must have $R \equiv 0$. Q.E.D.

As an application, consider the following example, which seems to be due to Dan Burns: let $\Sigma$ be a compact Riemann surface with $g>1$ equipped with its $R \equiv-1$ metric, and let $M=S^{2} \times \Sigma$, where $S^{2}$ is given its $R \equiv+1$ metric. Then $M$ is conformally flat, ${ }^{2}$ since $R \equiv 0$ and $M$ is Kähler with either orientation-just reverse

\footnotetext{
${ }^{2}$ Marcos Dajczer has pointed out that, more generally, $S^{m} \times H^{l}$ (where $H^{l}$ is $l$-dimensional hyperbolic space) is conformally flat for suitably adjusted curvatures, since it naturally sits in the light cone of Minkowski $(m+l+2)$-space.
} 
the orientation of $\Sigma$ ! The same remark applies to any flat 2 -sphere bundle over $\Sigma$ with structure group $\mathrm{SO}(3)$.

Here is a sort of converse.

Proposition 3. Let $M$ be a compact Kähler self-dual spin-manifold of type zero. Then $M$ is isometrically diffeomorphic to one of the following:

(1) a K3 surface with a "Yau metric";

(2) a flat 4-torus modulo a finite group; or

(3) a flat 2-sphere bundle over a Riemann surface of genus $>1$ with the local product metric.

Proof. If the signature $\sigma=b^{+}-b^{-}$does not vanish, we may apply the index theorem to the Dirac equation, concluding that there is a harmonic spinor since the signature of the Dirac operator $\downarrow$ is $\sigma / 8$. But a harmonic spinor is parallel when $R \equiv 0$ because $\downarrow^{2}=\nabla^{*} \nabla+R / 4$; having a parallel spinor then implies that at least one spin bundle is flat, which in turn implies that the canonical bundle is trivial. One then concludes that $M$ is a $K 3$ surface or a torus-the latter actually being excluded by the assumption that $\sigma \neq 0$. For details, see [3].

If $\sigma=0$ one may instead use the fact [4] that

$$
\int\left\|C^{+}\right\|^{2} d \text { vol }=12 \pi^{2} \sigma .
$$

Hence in this case $M$ is conformally flat. Since $b^{-}=b^{+} \neq 0, M$ is also Kähler with respect to the opposite orientation by Proposition 1; the holonomy is now subgroup of $U(1) \times U(1)$ and $M$ locally splits as a Riemannian product $\Sigma_{1} \times \Sigma_{2}$ of oriented surfaces. But since the scalar curvature of $M$ vanishes, the scalar curvatures of $\Sigma_{1}$ and $\Sigma_{2}$ are equal and opposite. Hence $M$ is either flat, yielding (2),or one of these foliations is by surfaces of constant positive curvature. These leaves are compact by Myers' theorem, and hence are 2-spheres by Gauss-Bonnet. But a foliation by compact, simply connected leaves has a manifold $\Sigma$ as its leaf space; in our case $\Sigma$ is given a constant negative curvature metric by the transverse foliation. This yields alternative (3). Q.E.D.

What can we say more generally about the type zero case when $w_{2} \neq 0$ ? Assuming that $b^{-} \neq 0$, we have a conjugate-oriented Kähler surface with $b^{-}=1+2 p_{g}$, where $p_{g}=h^{2,0}$ is the geometric genus; moreover, our Weitzenböck formula told us that all closed anti-self-dual forms are parallel, so $b^{-} \leqslant 3$ and $p_{g} \leqslant 1$ with equality only if the canonical bundle is trivial. For $w_{2} \neq 0$, this yields $p_{g}=0$.

Since the Ricci form is self-dual-i.e. anti-self-dual with respect to the complex orientation-we have $c_{1}^{2} \leqslant 0$ with equality iff $M$ is Ricci flat. By Yau's solution [5] of the Calabi conjecture, the latter class of manifolds consists precisely of those Kähler surfaces such that some power of the canonical bundle is trivial; these are covered by $K 3$ surfaces or 4-tori, and are called, respectively, Enriques surfaces and hyperelliptic surfaces [6].

Now an earlier theorem of Yau [7] asserts that a Kähler surface of nonnegative scalar curvature is either a blowup of either a ruled surface or $\mathbf{C P}_{2}$, or else has torsion first Chern class. We now apply this to get less precise information about the 
remaining cases $\left(p_{g}=0, c_{1}^{2}<0\right.$, and $\left.w_{2} \neq 0\right)$, concluding that the remaining possibilities are blowups of $\mathbf{C P}_{2}$ and ruled surfaces. We also have a lower bound on the number of points that must be blown up in order to arrange that $c_{1}^{2}<0$; the Riemannian-Roch theorem says $12\left(1-q+p_{g}\right)=c_{1}^{2}+c_{2}$, and hence $12(1-q)<$ $\chi$. If $b_{1}=2 q=0$, this then asserts that, with the conjugate orientation, $b^{+}>9$, and hence $M$ is diffeomorphic to $m \mathbf{C P}_{2} \# \overline{\mathbf{C P}}_{2}$ (i.e. $\mathbf{C P}_{2}$ with $m$ points blown up, given the conjugate orientation) for $m>9$.

Combining these remarks with the work of Donaldson and Freedman [8] we get the following

THEOREM. Let $M$ be a connected simply connected compact self-dual 4-manifold. Then one of the following must hold

(a) $M$ is conformally isometric to $S^{4}$,

(b) $M$ is conformally isometric to a conjugate-oriented Ricci-flat $K 3$ surface,

(c) $M$ is homeomorphic to $m \mathbf{C P}_{2}, m>0$,

(d) $M$ is diffeomorphic to $m \mathbf{C P}_{2} \# \overline{\mathbf{C P}}_{2}, m>9$,

(e) $M$ is type negative.

It remains to be seen whether possibilities (d) and (e) actually occur.

Acknowledgment. The author is indebted to N. Hitchin, R. Schoen, D. Burns, C. Boyer, B. Lawson, M. Micallef, M. Dajczer, and A. Vitter for a variety of helpful conversations and remarks.

POSTCRIPT. It has come to the author's attention that the $R>0$ version of Proposition 1 occurs in J. P. Bourgignon's paper, Les variétés de dimension 4 à signatures non-nulle dont la courbure est harmonique sont d'Einstein, Invent. Math. 63 (1981), 263-286, and that a different proof of Proposition 2 may be found in A. Derdzinski's article, Self-dual Kähler manifolds and Einstein manifolds of dimension four, Compositio Math. 49 (1983), 405-433.

\section{BIBLIOGRAPHY}

1. N. Hitchin, private communication; Y. S. Poon, D. Phil. Thesis, Oxford, 1985.

2. R. Schoen, Conformal deformation of metrics to constant scalar curvature, J. Differential Geom. 20 (1984), 479-495.

3. N. Hitchin, Harmonic spinors, Adv. Math. 14 (1974), 1-55.

4. M. Atiyah, N. Hitchin, and I. M. Singer, Self-duality in four-dimensional Riemannian geometry, Proc. Roy. Soc. London. Ser. A 362 (1978), 425-461.

5. S. T. Yau, On the Ricci curvature of a compact Kähler manifold, Comm. Pure. Appl. Math. 31 (1978), $339-411$.

6. P. Griffiths and J. Harris, Principles of algebraic geometry, Wiley, 1978.

7. S. T. Yau, On the curvture of compact Hermitian manifolds, Invent. Math. 25 (1974), 213-239.

8. D. Freed and K. Uhlenbeck, Instantons and four-manifolds, Springer-Verlag, 1985.

Department of Mathematics, State University of New York, Stony Brook, New York 11794 